\title{
Immunoreactivity to Meissner corpuscles and dermal nerves in a bullous arthropod bite reaction
}

\author{
Ana Maria Abreu Velez ${ }^{1}$, Alejandra Maria Jiménez-Echavarria², Michael S. Howard ${ }^{1}$ \\ ${ }^{1}$ Georgia Dermatopathology Associates, Atlanta, Georgia, USA, ${ }^{2}$ Dermatology Resident, Hospital das Clinicas da Facultad \\ de Medicina de la Universidad de São Paulo (HCFMUSP), Sao Paulo, Brazil
}

Corresponding author: Ana Maria Abreu Velez, M.D, Ph.D., E-mail: abreuvelez@yahoo.com

Abbreviations: Hematoxylin and eosin (H\&E), periodic acid Schiff (PAS), immunohistochemistry (IHC), direct immunofluorescence (DIF), 4,6-diamidino-2-phenylindole (DAPI), glial fibrillary acidic protein (GFAP), antibody to protein gene product 9.5 (PPG 9.5), Meissner corpuscle (MC).

Sir,

A clinical pruritus and inflammation is present following most insect bites. Here we document immunoreactivity to Meissner corpuscles and dermal nerves following arthropod bites.

Case: A 67 year old male presented with small, itchy red bumps, and recalled recent insect bites (Fig. la). We examined his skin biopsies utilizing hematoxylin and eosin (H\&E) and direct immunofluorescence (DIF). These tests and the clinical data demonstrated evidence of insect bites. Following the diagnosis of arthropod bites, the patient was instructed to wash the lesions with soap and water, apply cool compresses, use antihistamines to relieve itching and acetaminophen for pain relief. In addition, $1 \%$ Hydrocortisone cream was prescribed, and the patient reported symptomatic relief.

Our hematoxylin and eosin (H\&E), periodic acid Schiff (PAS) and multiple fluorochrome direct immunofluorescence (DIF) procedures were performed as previously described $[1,2]$. For nerve co-localization, we utilized $\mathrm{Cy}_{3}$ conjugated anti-glial fibrillary acidic protein (GFAP) antibody from Sigma-Aldrich (Saint Louis, Missouri, USA). We also used Texas red conjugated Protein Gene Product 9.5 antibody (PPG 9.5) from Abcam (Cambridge, Massachusetts, USA) and performed nuclear counterstaining with 4,6-diamidino-2-phenylindole (DAPI; Pierce, Rockford, Illinois, USA). All samples were consistently run with positive and negative controls. We classified our DIF findings as negative $(-)$, weakly positive $(+)$, positive $(++/+++)$ and strongly positive $(++++)$. H\&E staining showed subepidermal blistering, with a moderately florid superficial and perivascular infiltrate of lymphocytes, histiocytes and eosinophils; these findings were consistent with a bullous arthropod bite reaction (Fig. lb). The multicolor DIF showed reactivity to Meissner corpuscles (MCs) and dermal nerves under the bite, using neural markers colocalizing with antibodies to human Complement/C3c and fibrinogen (Figs. lc and d). The PAS stain showed positive staining of basement membrane areas around the blister.

Overall, our DIF displayed IgG (+, punctate staining in epidermal stratum spinosum); IgA, IgM, IgD, IgE, Complement/Clq, Complement/C3 and Kappa light chains (all ++ , all with punctate staining on possible dermal cell junctions of an unknown nature; Complement/C3 $(+++$, on dermal grouped nerves; albumin $(++$, staining on several large deep dermal nerves) and fibrinogen $(++$, dermal perivascular, on dermal nerves and on MCs). Large deep dermal nerves also stained positive for PPG9.5; small dermal nerves and Meissner corpuscles stained positive for GFAP $(+++)$. In summary, we detected immunoreactivity to dermal nerves and MCs subjacent to the insect bite using multiple fluorochrome DIF.

Bites or stings from arthropods are common, and sometimes an allergic reaction occurs to toxins

\footnotetext{
How to cite this article: Abreu Velez AM, Jiménez-Echavarria AM, Howard MS. Immunoreactivity to Meissner corpuscles and dermal nerves in a bullous arthropod bite reaction. Our Dermatol Online. 2017;8(1):102-103.

Submission: 04.10.2016; Acceptance: 20.11.2016

DOI: 10.7241 /ourd.20171.28
} 




Figure 1: (a) A clinical photograph shows soft, erythematous papules (black arrows). (b) H\&E staining shows a subepidermal blister (black arrow) with some fibrin inside the blister. Below the blister, note the perivascular infiltrate around upper dermal blood vessels (blue arrow; 100X). (c) Positive DIF staining on a MC, using anti-human FITC-conjugated fibrinogen antibodies (green staining; white arrow), cell nuclei are counterstained with DAPI (light blue). (d) Multiple fluorochrome DIF staining, using anti-human FITC conjugated fibrinogen antibodies and demonstrating positive staining to a MC, and its neurovascular supplies (green staining; white arrow), colocalizing with Texas red conjugated antibodies against GFAP (pink staining; white arrow). The yellow arrow shows positivity to a separate dermal neurovascular package.

associated with the bite or sting. Some individuals have severe clinical reactions to the stings of bees, wasps, hornets, yellow jackets and or other insects [3]. These stings may require emergency treatment for anaphylactic reactions. Here we report, to our knowledge for the first time an arthropod bite showing reactivity to Meissner's corpuscles (MCs). MCs are a type of nerve ending in the skin, responsible for sensitivity to light touch. These corpuscles display the highest sensitivity for detecting vibrations, and are rapidly adaptive receptors [4]. Nerves and Meissner corpuscles colocalized with neural antibodies, as well as autoantibodies developed by the patient after the arthropod bite. We conducted a combined PubMed search for all publication years entering "arthropod bites" and the Mesh words "Meissner corpuscles"; we found no citations. We have previously reported nerve reactivity following a scabies mite infection. MCs are part of the purinergic signaling system; this system involves adenosine 5'-triphosphate and adenosine receptors, neurotransmission, exocrine and endocrine secretion and regulation of immune cell function [4].

The significance of our findings is unknown; we suggest further investigation into these findings.

\section{REFERENCES}

1. Abreu Velez AM, Klein AD, Howard MS. Autoreactivity to sweat glands and nerves in aclinical scabies infection. North Am J Med Sci. 2010;2:422-5.

2. Abreu Velez AM, Calle Isaza J, Howard MS. Immunofluorescence patterns in selected dermatoses, including blistering skin diseases utilizing multiple fluorochomes. North Am J Med Sci. 2015;7:397-402.

3. Singh S, Mann BK. Insect bite reactions. Indian J Dermatol Venereol Leprol. 2013;79:151-64.

4. Paré M, Elde R, Mazurkiewicz JE, Smith AM, Rice FL. The Meissner corpuscle revised: a multiafferented mechanoreceptor with nociceptor immunochemical properties. J Neurosci. 2001;15;21:7236-46.

Copyright by Ana Maria Abreu Velez, et al. This is an open-access article distributed under the terms of the Creative Commons Attribution License, which permits unrestricted use, distribution, and reproduction in any medium, provided the original author and source are credited.

Source of Support: Georgia Dermatopathology Associates, Atlanta, Georgia, USA, Conflict of Interest: None declared. 